\title{
Performance Analysis of Energy Consumption of Smartphone Running Mobile Hotspot Application
}

\author{
Yun Won Chung \\ School of Electronic Engineering, Soongsil University, 511 Sangdo-dong, Dongjak-gu, Seoul, 156-743, Korea
}

\begin{abstract}
Recently, smartphones are widely used due to the popularity of Internet, and they are equipped with both Wi-Fi and cellular radio interfaces generally. In smartphone, although Wi-Fi interface is generally used for supporting higher rate data session of the smartphone itself, it can be also used to support connectivity to other nearby wireless devices with Wi-Fi interface only, such as laptop. This is achieved by mobile hotspot application of smartphones, where smartphone acts as an access point (AP) for nearby wireless devices using Wi-Fi interface. By using the Wi-Fi interface, nearby wireless devices transmit data to smartphone, and smartphone uses its cellular interface to deliver the data to wireless network. However, data transmission of nearby wireless devices using mobile hotspot application consumes significant battery energy of smartphone since Wi-Fi interface of the smartphone should be awaken always and data session of nearby wireless devices should be processed with both Wi-Fi and cellular interfaces of smartphone. In this paper, we develop an analytical model of energy consumption of smartphone running mobile hotspot application and analyze the effect of various parameters on the energy consumption of smartphone. The result of this work can be used to propose an enhanced mobile hotspot scheme by giving insight on the energy consumption.
\end{abstract}

Keywords: Energy consumption, smartphone, mobile hotspot, performance analysis

\section{Introduction}

Recently, smartphones are widely used due to the popularity of Internet, and they are equipped with both Wi-Fi and cellular radio interfaces generally [1]. Wi-Fi interface is used for supporting higher data rate services with cheap price, but the coverage of Wi-Fi is limited and no seamless handover is supported [2]. On the other hand, cellular interface is used to provide continuous connectivity and seamless handover, although it has a higher access cost. In smartphone, although Wi-Fi interface is generally used for supporting higher rate data session of the smartphone itself, it can be also used to support connectivity to other nearby wireless devices with Wi-Fi interface only, such as laptop. This is achieved by mobile hotspot application of smartphones, where smartphone acts as an access point (AP) for nearby wireless devices using Wi-Fi interface [3], [4]. By using the Wi-Fi interface, nearby wireless devices transmit data to smartphone, and smartphone uses its cellular interface to deliver the data to wireless network, as shown in Fig. 1. We will call the nearby wireless devices within the mobile hotspot area of a smartphone as mobile hotspot client in this paper.

To act as an AP for nearby wireless devices, smartphone should awake its Wi-Fi interface always and power saving mode should not be enabled. Also, the data received from nearby wireless devices using the Wi-Fi interface of smartphone should be transmitted via its cellular interface of the smartphone, too. Therefore, significant battery energy is consumed to run mobile hotspot application. In order to reduce the energy consumption of smartphones running mobile hotspot application, a few works have been carried out recently [3], [4].

\footnotetext{
* Manuscript received July 24, 2012; revised August 26, 2012.

Corresponding author. Tel.: +82-2-820-0908; fax: +82-2-821-7653; E-mail address: ywchung@ssu.ac.kr.
} 


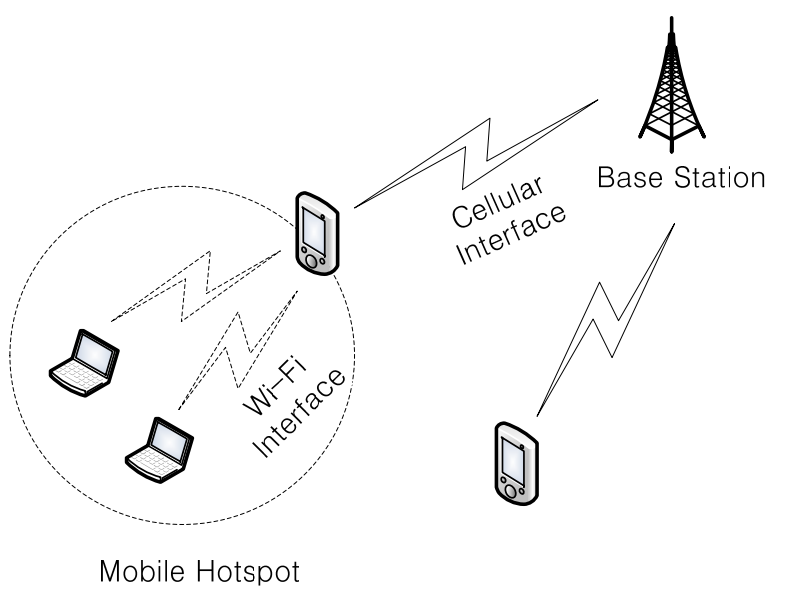

Fig. 1. Mobile hotspot scenario.

In [3], since cellular network interface consumes extra energy until a predefined timer expires, even though the final packet is transmitted, the authors proposed to gather all the related data and send the data in a single burst in order to minimize the unnecessary extra energy consumption after the completion of packet transmission. Also, the authors proposed reverse-infrastructure mode, where Wi-Fi clients of smartphone serve as a gateway and thus, smartphone can operate in low-power power saving mode.

In [4], the authors carried out experiments to measure energy consumption of smartphone with LTE and Wi-Fi interfaces. Based on the measurement result, the authors found out that LTE and Wi-Fi interfaces of smartphone running mobile hotspot application consume significant battery energy. Then, they proposed energy efficient scheduling by coordinating power saving techniques of both Wi-Fi and LTE in an integrated way. In the proposed scheduling scheme, LTE sleep pattern and Wi-Fi sleep pattern are combined efficiently to achieve maximum power saving for smartphone in idle mode. Also, for smartphone in connected mode where both Wi-Fi and LTE are in active mode, LTE long discontinuous reception (DRX) and short DRX modes are synchronized with Wi-Fi sleep pattern for downlink data. By doing this scheme, Wi-Fi clients can sleep as much as possible and thus, power consumption of smartphone is reduced. For uplink data from Wi-Fi interface, smartphone aggregates data from multiple Wi-Fi clients, buffers the data, and transmit the data using single LTE on duration for background traffic.

Although the works in [3], [4] analyzed the energy consumption of smartphone running mobile hotspot application, most works have been carried out via simulation and little work has been carried out for analytical modeling and performance analysis of energy consumption of smartphone supporting mobile hotspot application, to the best of our knowledge. However, the analytical modeling on energy consumption of smartphone running mobile hotspot application is essential to analyze the effect of various parameters on the energy consumption of smartphone and propose an enhanced energy management scheme based on the performance analysis results. Therefore, we develop an analytical modeling for energy consumption of smartphone running mobile hotspot application for nearby wireless devices. The result of this work can be used to propose an enhanced mobile hotspot scheme by giving insight on the energy consumption.

The remainder of this paper is as follows: Section 2 develops an analytical modeling for the energy consumption of smartphone running mobile hotspot. Then, numerical examples are presented in Section 3 . Finally, Section 4 concludes this work and presents future work.

\section{Performance Analysis}

Fig. 2 shows network architecture with mobile hotspot and public hotspot within a cell of cellular network considered in this paper. Mobile hotspot clients are using their Wi-Fi interfaces to transmit their data to smartphone running mobile hotspot application. The data of mobile hotspot clients, which are received from Wi-Fi interface of the smartphone, are transmitted to wireless network, using the cellular 
interface of the smartphone. If a smartphone does not run mobile hotspot application and resides within the range of public hotspot, it uses its Wi-Fi interface to connect to public hotspot and sends its data using the Wi-Fi interface since it provides higher transmission rate with much cheaper price. On the other hand, if a smartphone is not within the public hotspot, it uses its cellular interface to transmit data to wireless network.

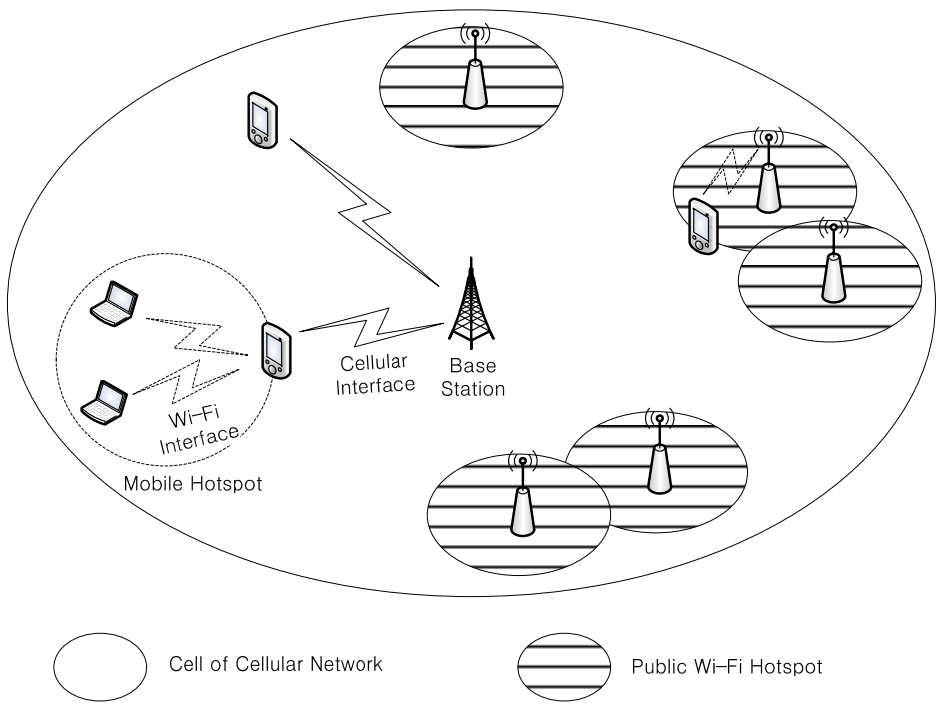

Fig. 2. Network architecture

For performance analysis of energy consumption of smartphone running mobile hotspot application, we have made the following assumptions:

- The ratio of the service coverage of public hotspot areas within a cell of cellular network over service coverage by a cell is assumed as $\alpha$.

- The ratio of the transmission rate of cellular interface over the transmission rate of Wi-Fi interface of a smartphone is assumed as $\beta$.

- The session arrival rate of a mobile hotspot client $i$ using Wi-Fi interface follows a Poisson distribution with parameter $\lambda_{i}^{W}$.

- The session duration of a mobile hotspot client $i$ using Wi-Fi interface follows an exponential distribution with parameter $\mu_{i}^{W}$.

- The session duration of a mobile hotspot client $i$ using cellular interface of a smartphone after received from the Wi-Fi interface of a smartphone follows an exponential distribution with parameter $\mu_{i}^{C}$.

- The number of mobile hotspot clients within mobile hotspot area of a smartphone is assumed as $N_{C}$.

- The session arrival rate of a smartphone user itself follows a Poisson distribution with parameter $\lambda_{S}$.

- The session duration of a smartphone itself using its Wi-Fi interface for session transmission follows an exponential distribution with parameter $\mu_{S}^{W}$.

- The session duration of a smartphone itself using its cellular interface for session transmission follows an exponential distribution with parameter $\mu_{S}^{C}$.

Based on the above assumptions, we derive energy consumption of smartphone per unit time when mobile hotspot application is either running or not running. For notational convenience, we denote the case where mobile hotspot application is not running by Scheme 1. The case where mobile hotspot application is running is denoted by Scheme 2. The energy consumption of smartphone per unit time when mobile hotspot application is not running, i.e., Scheme 1, is obtained as [5]:

$$
E_{1}=\frac{\lambda_{S}(1-\alpha)}{\mu_{S}^{C}} P_{\text {active }}^{C}+\left[1-\frac{\lambda_{S}(1-\alpha)}{\mu_{S}^{C}}\right] P_{\text {idle }}^{C}+\frac{\lambda_{S} \alpha}{\mu_{S}^{W}} P_{\text {active }}^{W}+\left[1-\frac{\lambda_{S} \alpha}{\mu_{S}^{W}}\right] P_{\text {idle }}^{W}
$$


where $P_{\text {active }}^{W i-F i}, P_{i d l e}^{W i-F i}, P_{\text {active }}^{\text {celluar }}$, and $P_{\text {idle }}^{\text {cellular }}$ are energy consumption of active Wi-Fi interface, idle Wi-Fi interface, active cellular interface, and idle cellular interface of smartphone, respectively. Also, the energy consumption of smartphone per unit time when mobile hotspot application is running, i.e., Scheme 2, is obtained as [5]:

$$
E_{2}=\sum_{i=1}^{N_{C}} \frac{\lambda_{i}}{\mu_{i}^{W}} P_{\text {active }}^{W}+\left[1-\sum_{i=1}^{N_{C}} \frac{\lambda_{i}}{\mu_{i}^{W}}\right] P_{\text {idle }}^{W}+\left(\sum_{i=1}^{N_{C}} \frac{\lambda_{i}}{\mu_{i}^{C}}+\frac{\lambda_{S}}{\mu_{S}^{C}}\right) P_{\text {active }}^{C}+\left[1-\sum_{i=1}^{N_{C}} \frac{\lambda_{i}}{\mu_{i}^{C}}-\frac{\lambda_{S}}{\mu_{S}^{C}}\right] P_{\text {idle }}^{C}
$$

\section{Numerical Examples}

For numerical examples, we assume the session arrival rate of a mobile hotspot client $i$ is independent and identically distributed, and thus, $\lambda_{i}=\lambda$ for all $i$ and $\mu_{i}^{W}=\mu$ for all $i$ are assumed for mathematical simplicity. Also, based on the assumption of $\beta$ as the ratio of the transmission rate of cellular interface over the transmission rate of Wi-Fi interface of a smartphone, $\mu_{i}^{C}=\beta \mu_{i}^{W}=\beta \mu$ and $\mu_{S}^{C}=\beta \mu_{S}^{W}$ are obtained since transmission time is inversely proportional to transmission rate of a wireless interface.

Fig. 3 shows the energy consumption for varying the values of $\alpha$ for $P_{\text {idle }}^{\text {cellular }}=0.125(\mathrm{~W}), P_{\text {active }}^{\text {cellular }}=1.254$ (W), $P_{\text {idle }}^{W i-F i}=1.15(\mathrm{~W}), P_{\text {active }}^{W i-F i}=1.65(\mathrm{~W}), \beta=0.2, N_{C}=5, \lambda_{S}=3, \mu_{S}^{C}=30, \mu_{S}^{W}=\mu_{S}^{C} / \beta, \lambda=3$, and $\mu=30$. As the figure shows, the energy consumption of Scheme 2 is irrelevant for varying the values of $\alpha$, since mobile hotspot clients always connect to smartphone by using mobile hotspot application and thus, it does not depend on the service coverage of public hotspot. However, the energy consumption of Scheme 1 decreases slightly as the value of $\alpha$ increases, since the probability of session of mobile hotspot clients being served by public hotspot increases as the value of $\alpha$ increases. Also, it is shown that Scheme 2 has much higher energy consumption than Scheme 1, which is about three times for a given parameter setting.

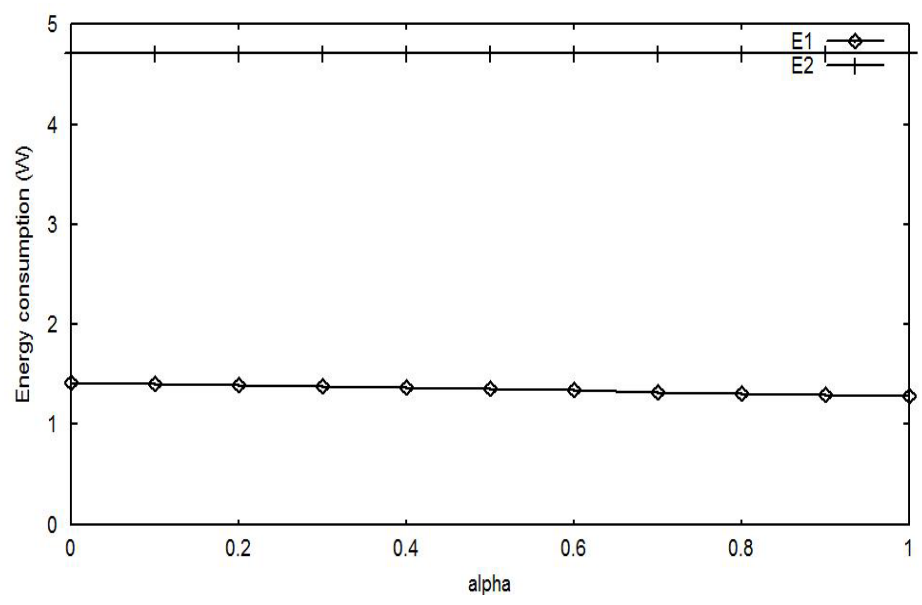

Fig. 3. Energy consumption for varying $\alpha$.

Fig. 4 shows the energy consumption for varying the values of $\beta$ for $P_{\text {idle }}^{\text {cellular }}=0.125(\mathrm{~W})$, $P_{\text {active }}^{\text {cellular }}=1.254(\mathrm{~W}), P_{\text {idle }}^{W i-F i}=1.15(\mathrm{~W}), P_{\text {active }}^{W i-F i}=1.65(\mathrm{~W}), \alpha=0.2, N_{C}=5, \lambda_{S}=3, \mu_{S}^{C}=30, \mu_{S}^{W}=\mu_{S}^{C} / \beta$, $\lambda=3$, and $\mu=30$. As the figure shows, the energy consumption of Scheme 2 decreases as the value of $\beta$ increases, since the larger value of $\beta$ means smaller transmission time in cellular interface for the same data session, which results in less energy consumption in cellular interface. However, the energy consumption of Scheme 1 increases slightly as the value of $\beta$ increases, since the transmission time in WiFi interface increases as the value of $\beta$ increases for a given value of $\mu_{S}^{C}$, which results in more energy consumption in Wi-Fi interface. 


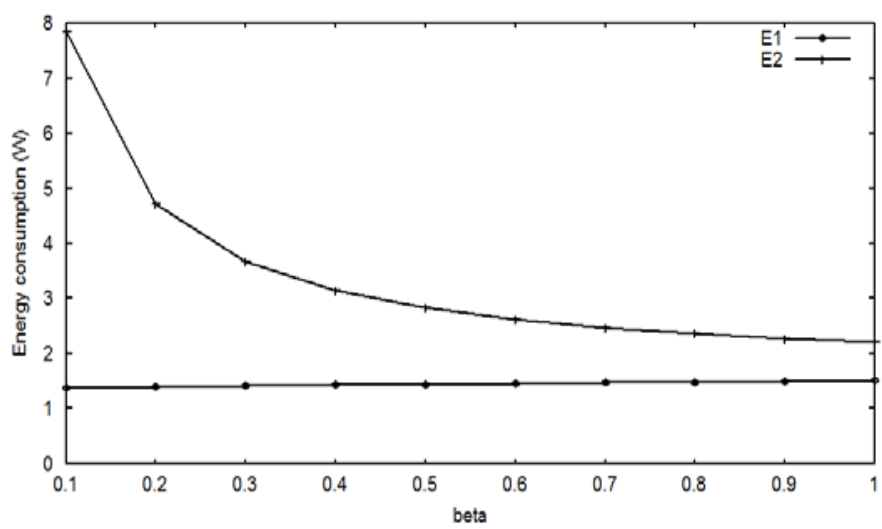

Fig. 4. Energy consumption for varying $\beta$.

Fig. 5 shows the energy consumption for varying the values of $\rho=N_{C} \lambda / \mu$ for $P_{\text {idle }}^{\text {cellular }}=0.125(\mathrm{~W})$, $P_{\text {active }}^{\text {cellular }}=1.254(\mathrm{~W}), P_{\text {idle }}^{W i-F i}=1.15(\mathrm{~W}), P_{\text {active }}^{W i-F i}=1.65(\mathrm{~W}), \alpha=0.2, \beta=0.2, N_{C}=5, \lambda_{S}=3, \mu_{S}^{C}=30$, and $\mu_{S}^{W}=\mu_{S}^{C} / \beta$. As the figure shows, the energy consumption of Scheme 2 increases as the value of $\rho$ increases, since the larger value of $\rho$ means more transmission time in both cellular and Wi-Fi interfaces of Scheme 2, which results in more energy consumption in both cellular and Wi-Fi interfaces. However, the energy consumption of Scheme 1 does not depend on the value of $\rho$.

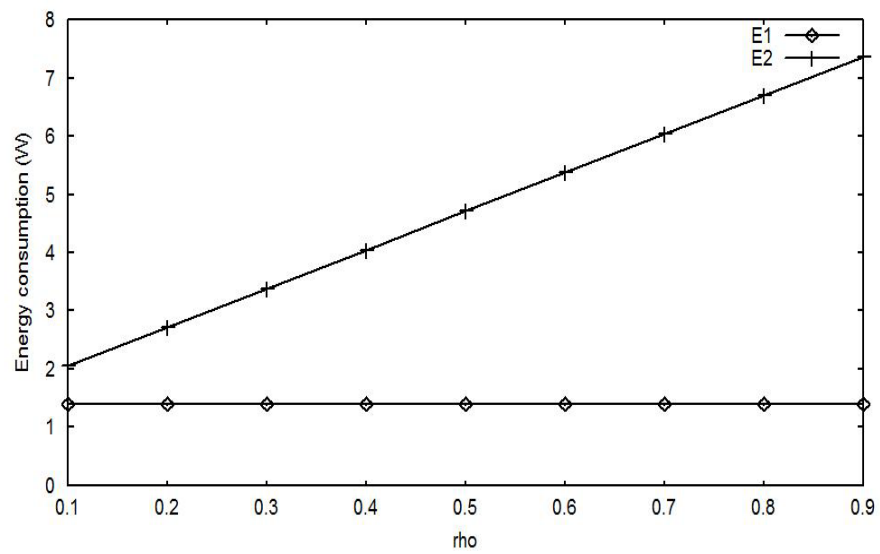

Fig. 5. Energy consumption for varying $\alpha$.

\section{Conclusion And Future Work}

In this paper, we developed an analytical modeling for the analysis of the energy consumption of smartphone per unit time when mobile hotspot is either running or not running. Then, we analyzed the effect of various parameters on the energy consumption. From the numerical results, it was concluded that the energy consumption of smartphone running mobile hotspot application is very sensitive to both the ratio of the transmission rate of cellular interface over the transmission rate of Wi-Fi interface of a smartphone and the activity of aggregated sessions of mobile hotspot clients. It was also concluded that the energy consumption of smartphone running mobile hotspot application has much higher values than that of smartphone without running mobile hotspot application. In our future work, we will extend our current work by developing a more completed analytical model based on more practical assumptions on network architecture, and mobility and traffic characteristics of mobile clients and smartphone. Also, based on performance analysis results, an improved energy management scheme for smartphone running mobile hotspot application will be proposed and analyzed. 


\section{Acknowledgements}

This research was supported by Basic Science Research Program through the National Research Foundation of Korea (NRF) funded by the Ministry of Education, Science and Technology (MEST) (20120002453).

\section{References}

[1] Friedman R, Kogan A, Krivolapov Y. On power and throughput tradeoffs of WiFi and bluetooth in smartphones. In: Proc. of IEEE INFOCOM, 2011: 900-908.

[2] Chung YW, Lee SW. Modeling and performance analysis of power efficient multi-tier location management in interworked WLAN and cellular network. Elsevier Mathematical and Computer Modelling; doi:10.1016/j.mcm.2012.01.007.

[3] Sharma A, Navda V, Ramjee R, Padmanabhan VN, Belding EM. Cool-tether: energy efficient on-the-fly WiFi hot-spots using mobile phones. Presented at: IEEE CoNEXT 2009.

[4] Keshav K, Indukuri VR, Venkataram P. Energy efficient scheduling in 4G smart phones for mobile hotspot application. In: Proc. of 2012 National Conference on Communications (NCC), 2012:1-5.

[5] Ross SM. Stochastic Processes. John Wiley \& Sons; 1996. 